\title{
ORIGINAL ARTICLE Reliability of the electrical perceptual threshold and Semmes-Weinstein monofilament tests of cutaneous sensibility
}

\author{
PH Ellaway and M Catley
}

\begin{abstract}
Study design: Prospective experimental.
Objectives: To compare the reliability and repeatability of the electrical perceptual threshold (EPT) and Semmes-Weinstein monofilament (SWM) tests for cutaneous sensibility. EPT and SWM tests have potential as quantitative and sensitive adjuncts to the American Spinal Injuries Association (ASIA) Impairment Scale (AIS) assessment of spinal cord injury (SCI).

Setting: London, UK.

Methods: EPT and SWM tests were carried out on 40 neurologically healthy individuals (20 male). One examiner carried out all the tests. Each individual was examined for EPT and SWM sensitivity at ASIA key points on four dermatomes (C4, T1, T6, L4) on both sides of the body. The tests were repeated after an interval of approximately 1 week. Intra-rater reliability was determined using intraclass correlation coefficients (ICC). Repeatability was determined using the method of Bland and Altman.

Results: There were no significant differences in the mean values of EPT or SWM between assessments for any dermatome. Significant difference in mean values for both EPT and SWM were observed between some dermatomes. ICC ranged from 0.67 to 0.81 for the EPT and from 0.46 to 0.61 for the SWM. Higher ICC for the EPT compared with the SWM was again revealed when male and female subjects were assessed separately. Correlation between EPT and SWM was weak or (largely) absent.
\end{abstract}

Conclusion: EPT has better reliability than SWM in healthy subjects. However, as both tests have the potential to add sensitivity and resolution to the AIS assessment, a further comparison of their repeatability in $\mathrm{SCl}$ is warranted.

Spinal Cord (2013) 51, 120-125; doi:10.1038/sc.2012.96; published online 4 September 2012

Keywords: quantitative sensory tests; sensory perceptual threshold; electric current; monofilaments

\section{INTRODUCTION}

The International Standards for Neurological Classification of Spinal Cord Injury (ISNSCI) is the established clinical neurologic practice for the characterisation of spinal cord injury (SCI). The ISNCI incorporates the current gold standard for neurological assessment of SCI, the American Spinal Injuries Association (ASIA) Impairment Scale (AIS). ${ }^{1}$ These scales were initially developed primarily to classify the neurologic deficit following a SCI, but are now widely used as outcome measures to monitor any change in the condition of a SCI during natural recovery or treatments designed to promote recovery of function. The AIS provides a reliable clinical assessment tool, but it is recognised that additional quantitative assessments will be needed to meet the precision required for clinical trials. ${ }^{2}$

The ASIA component light touch and pin prick sensory assessments have a number of limitations. Both sensory scores use ordinal rather than quantitative scales. Each dermatome is scored simply as either normal, absent or abnormal (including both heightened and lowered sensitivity). It is recognised that there is also is a strong component of subjectivity in the assessments. Greater objectivity and more detailed valuation can be achieved by employing various quantitative sensory tests for the assessment of preserved sensory function in SCI. ${ }^{3}$ Improved outcome measures should allow both for detection of betterment or worsening of the condition of SCI, and they should be capable of detecting change at a single vertebral level of the spinal cord. An emerging test that has the potential to achieve these criteria is the electrical perceptual threshold test (EPT). ${ }^{4}$

The EPT method uses incrementing electrical stimulation and the method of limits to determine threshold. It has been validated against the AIS sensory grading in $\mathrm{SCI}^{5,6}$ and undergone repeatability evaluation for inter and intra-rater trials in SCI. ${ }^{7}$ Good reliability has now been confirmed in control subjects, ${ }^{8}$ and validation provided against the AIS for sensory evaluation ${ }^{6}$ and dermatomal somatosensory evoked potentials in SCI. ${ }^{9}$ The EPT test shows good sensitivity to change over time in dermatomes at and below the sensory level of SCI during natural recovery ${ }^{10}$ Finally, the EPT appears to show specificity for the light touch (dorsal column) pathway rather than the anterolateral spinothalamic tract. ${ }^{7,9,11}$

Improved sensitivity of quantitative sensory testing will aid more accurate diagnosis of spinal cord-lesioned individuals, allowing more appropriately targeted rehabilitation and better management of these patients. Additionally, with the commencement of translational clinical trials for the repair of SCI, advancement of quantitative sensory testing ${ }^{3}$ is now necessary to detect the possibly limited changes likely to result from initial efforts. ${ }^{2}$ Semmes-Weinstein monofilaments (SWM) are a well-established test of mechanical cutaneous sensibility that have been widely used in the assessment 
of peripheral neuropathy but, in respect of SCI, have mainly been employed to investigate neuropathic pain ${ }^{12}$ rather than light touch. ${ }^{13}$ It is particularly relevant at this time to evaluate the performance of SWM against EPT as the SWM have recently been proposed as test of cutaneous sensibility when evaluating hand function in SCI. ${ }^{14}$ In terms of application, we have found previously that EPT is well tolerated by subjects, not time consuming to perform and requires apparatus of relatively modest cost. , $7^{7}$ The present study allows us to confirm these attributes also apply to the use of SWM. No adverse effects on subjects for either procedure have been reported in the literature. This paper sets out to examine the sensitivity and compare the reliability of these two tests of cutaneous sensibility in healthy individuals. Such evaluation is necessary ahead of further applications in SCI to establish their respective reliability for dermatomes in the absence of any impact likely caused by SCI.

\section{MATERIALS AND METHODS}

The study was approved by Imperial College Research Ethics Committee. All volunteers were given a written information sheet and a verbal explanation of the procedure before signing a consent form.

\section{Subjects}

Perceptual thresholds to cutaneous electrical stimulation and mechanical sensitivity to monofilaments (Semmes-Weinstein) were measured in 40 healthy subjects with no known neurological deficits (20 male subjects, aged 23-68 years, median age 35 ; 20 female subjects, aged 21-61 years, median age 29).

\section{Study design}

A prospective test-retest study to measure reliability of EPT and SWM over time and additionally aimed to assess the degree of correlation between the two procedures. Each subject was tested on two occasions by the same assessor. The same assessor was employed for all the tests. Experience of the EPT and SWM test procedures was gained by the assessor over a period of 1 week before the commencement of the study. Consistent, repeat measures were obtained by the assessor after approximately 2-3 days training. During the study, the two sets of tests were performed approximately 1-2 weeks apart with a median interval of 8 days. Subjects were required to lie supine on a clinical examination couch in a quiet, warm room. Clothing was adjusted, as required, to gain access to dermatomes C4, T1, T8 or L4 that were selected for analysis. Two of these dermatomes were selected as they have, respectively, the lowest (T1) and highest (L4) EPT values. ${ }^{6}$ As these dermatomes are on limbs, we also selected dermatomes on the shoulder (C4) and abdomen (T8) to have a fairly broad representation of dermatomes over the body.

\section{Outcome measures}

The technique used to determine EPT was that previously described by King et al., ${ }^{7}$ using a computer-controlled stimulator. Electrical stimulation was delivered using a constant current electrical stimulator Digitimer DS5 (Digitimer, Welwyn Garden City, UK) controlled using a data acquisition card (USB-6211, National Instruments, Austin, TX, USA) connected to a laptop PC running an in-house programme. Square wave (monophasic) current pulses (width of $0.5 \mathrm{~ms}$ ) were delivered at a repetition rate of $3 \mathrm{~Hz}$. The maximum current allowable was preset at $10 \mathrm{~mA}$, a level approximately three times greater than the highest thresholds found previously in healthy individuals. ${ }^{6}$ Stimuli were applied between an adhesive cathode electrode (Arbo Neonatal ECG Blue, $22 \mathrm{~mm}$ diameter conducting surface contact) placed over the key ASIA sensory point of a dermatome as delimited in the International Standards of Neurological Classification of Spinal Cord Injury $(\text { ISNCSCI })^{1}$ and a metal anodal electrode $(4 \times 2.5 \mathrm{~cm})$ located on the forearm approximately $10-15 \mathrm{~cm}$ proximal to the wrist. Above threshold stimulation was described as a light, tapping sensation at the location of the cathode. Using the method of limits, the subjects were asked to report immediately when they could first feel stimulation as the intensity was raised from $0 \mathrm{~mA}$ and then again when sensation disappeared as intensity was lowered. The rates of ascent and descent were set at $0.1 \mathrm{~mA}$ per step increments after every third stimulus. Reported occurrence and disappearance of sensation by the subject were logged by operator key strokes. The computer software continued to increase stimulator output by three increments of $0.1 \mathrm{~mA}$ before reversing the change in intensity. The procedure was repeated three times and the EPT (mA) was calculated as the mean of the intensities logged as sensation just disappeared on lowering the stimulus strength.

Sensory evaluation using the SWM was carried out using a graded set of 20 monofilaments (Touch Test Sensory Evaluators, Stoetling Co., Wood Dale, IL, USA). The assessor started each test on any dermatome with a stiffness of filament regarded as indicative of the upper end of the range for normal skin sensitivity for the hand or dorsum of the foot (filament 2.83). The filament was applied on five separate occasions at random intervals of time with the subject's eyes closed. Filaments were applied vertically to the surface of the skin and force applied until the filament just started to bend. The application was continued for approximately $1 \mathrm{~s}$. The assessor noted the number of times the subject reported sensation to each application. Depending upon the result, that is, whether the subject could or could not detect the stimulus, filaments of lesser or greater stiffness were then applied. The weakest filament that gave a response rate of at least three out of five applications was accepted as threshold. The threshold was recorded as the $\log _{10}(10 \times$ force in $\mathrm{mg})$ exerted by that monofilament, as listed by the manufacturer.

The order in which the EPT and SWM tests were conducted was randomised for both the initial and repeat assessments.

\section{Analysis and statistics}

Data were analysed using Sigmaplot and Sigmastat (Systat Software Inc., Chicago, IL, USA). SWM and EPT measures from repeated assessment by the same rater for each of the levels were analysed individually using two-way repeated measures of analysis of variance (ANOVA) with the within-subject effects of assessment (first, second) and dermatome (C4, T1, T8 and L4). When the ANOVA revealed an interaction between assessment and dermatome, post hoc multiple pairwise comparisons were made with the Bonferroni $t$-test. The SWM and EPT data were also analysed individually for rater reliability by calculating the two-way random effect model ANOVA intra-class correlation coefficient (ICC) for single measure reliability. The 95\% limits of agreement were calculated and plotted according to the method described by Bland and Altman. Pearson's product moment correlation $(r)$ was calculated to compare SWM and EPT values. Significance was set at $P<0.05$ for all statistical analyses.

\section{RESULTS}

\section{Electrical perceptual threshold}

The repeated measure ANOVA for all subjects revealed no significant difference in the mean values of EPT between the two assessments for any dermatome. However, the difference in the mean values among the different levels of dermatome was greater than would be expected by chance $\left(\mathrm{F}_{3,639}=106.9, P=<0.001\right)$. Distributions of EPT measures for the two separate assessments are shown in Figure 1 and outcomes of post hoc all pairwise multiple comparisons are presented in Table 1. Significant differences in EPT were revealed between all but the $\mathrm{C} 4$ and $\mathrm{T} 1$ dermatomes.

\section{Semmes-Weinstein monofilaments}

The repeated measure ANOVA for all subjects revealed no significant difference in the mean values of SWM between the two assessments for any dermatome. However, the difference in the mean values among the different levels of dermatome was greater than would be expected by chance $\left(\mathrm{F}_{3,639}=66.3, P=<0.001\right)$ after allowing for effects of differences in assessment. Distributions of SWM measures for the two separate assessments are shown in the Figure 1 and outcomes of post hoc all pairwise multiple comparisons are presented in Table 1. Significant differences in SWM thresholds were revealed 


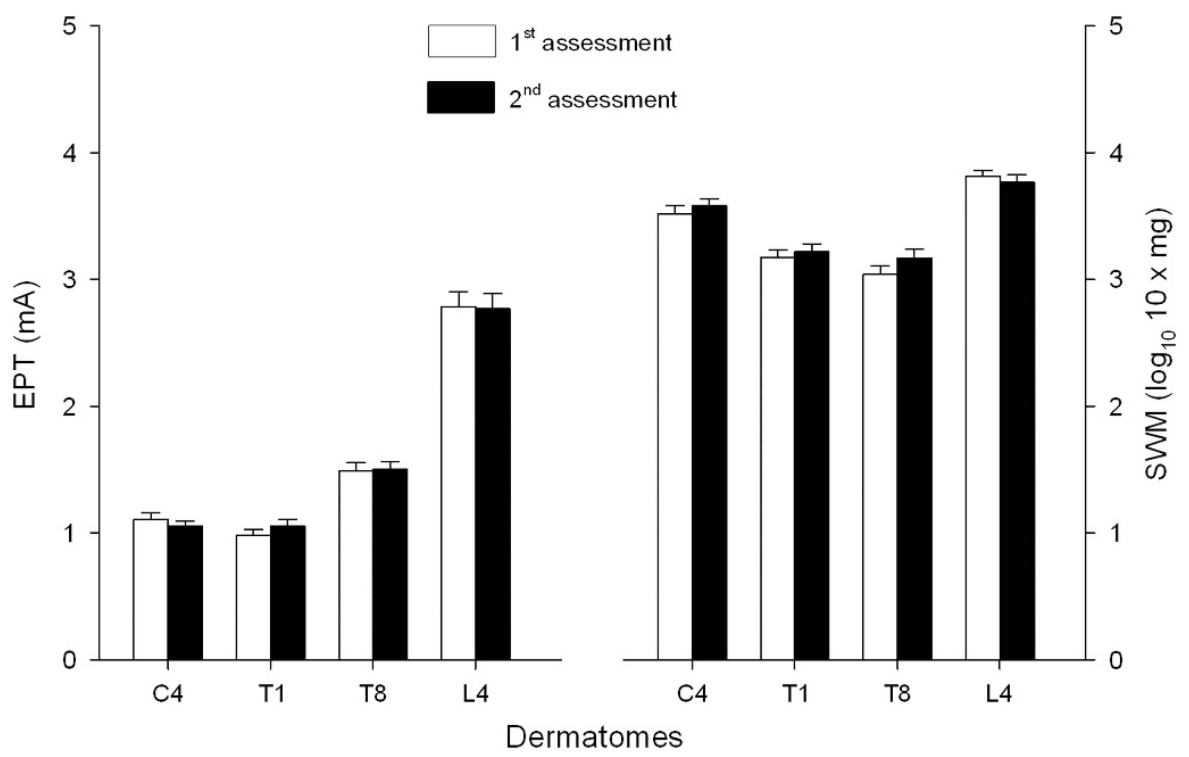

Figure 1 Mean (+s.e.) values for EPT (left) and SWM (right) thresholds for the first (open bars) and second (filled bars) assessments for the four dermatomes $\mathrm{C} 4, \mathrm{~T} 1, \mathrm{~T} 8$ and $\mathrm{L} 4$ for all 40 subjects.

Table 1 Post hoc multiple pairwise comparisons between dermatomes with Bonferroni $t$-test following significant interactions revealed by two-way repeated measures of ANOVA with the within-subject effects of assessment (first, second) and dermatome (C4, T1, T8 and L4)

\begin{tabular}{|c|c|c|c|c|c|c|c|c|}
\hline \multirow[t]{3}{*}{ Dermatomes } & \multicolumn{4}{|c|}{ SWM } & \multicolumn{4}{|c|}{$E P T$} \\
\hline & \multicolumn{2}{|c|}{ Assessment 1} & \multicolumn{2}{|c|}{ Assessment 2} & \multicolumn{2}{|c|}{ Assessment 1} & \multicolumn{2}{|c|}{ Assessment 2} \\
\hline & Diff of means & $P$ & Diff of means & $P$ & Diff of means & $P$ & Diff of means & $P$ \\
\hline C4 vs T1 & 0.34 & $<0.001$ & 0.36 & $<0.001$ & 0.12 & Insignificant & 0.002 & Insignifican \\
\hline C4 vs T8 & 0.48 & $<0.001$ & 0.41 & $<0.001$ & 0.38 & 0.002 & 0.45 & $<0.001$ \\
\hline C4 vs L4 & 0.3 & $<0.001$ & 0.18 & Insignificant & 1.68 & $<0.001$ & 1.78 & $<0.001$ \\
\hline T1 vs T8 & 0.13 & Insignificant & 0.05 & Insignificant & 0.51 & $<0.001$ & 0.45 & $<0.001$ \\
\hline T1 vs L4 & 0.64 & $<0.001$ & 0.55 & $<0.001$ & 1.80 & $<0.001$ & 1.71 & $<0.001$ \\
\hline T8 vs L4 & 0.78 & $<0.001$ & 0.6 & $<0.001$ & 1.29 & $<0.001$ & 1.27 & $<0.001$ \\
\hline
\end{tabular}

Abbreviations: ANOVAs, analysis of variance; EPT, electrical perceptual threshold; SWM, Semmes-Weinstein monofilament.

ANOVA's conducted independently for SWM and EPT measures.

between some dermatomes but not between $\mathrm{T} 1$ and $\mathrm{T} 8$ (either assessment) or between C4 and L4 (second assessment).

Test-retest reliability of SWM and EPT

The ICC comparing the two assessments (all subjects) for each dermatome for the SWM and for the EPT are presented in Table 2. ICC ranged from 0.46 to 0.61 for the SWM and from 0.67 to 0.81 for the EPT. Lower ICC for the SWM compared with the EPT was again revealed when male and female subjects were assessed separately. Mean ICC for the four dermatomes for males were SWM 0.57 (range $0.37-0.7$ ) and EPT 0.72 (range 0.58-0.81), and for females were SWM 0.54 (range 0.24-0.66) and EPT 0.67 (range 0.47-0.8).

The limits of agreement between first and repeat assessments for EPT and SWM are presented as Bland-Altman plots in Figure 2. There are only minor differences in the $95 \%$ limits between the different dermatomes for both the methods. There was no relationship between the size of the difference between assessments and the mean magnitude of a measure for any dermatome for either method.
Table 2 ICC for repeated measures of EPT and SWM by the same rater on two occasions

\begin{tabular}{llr}
\hline \multirow{2}{*}{ Dermatome } & \multicolumn{2}{c}{ ICC } \\
\cline { 2 - 3 } & EPT & SWM \\
\hline C4 & 0.67 & 0.46 \\
T1 & 0.73 & 0.61 \\
T8 & 0.74 & 0.55 \\
L4 & 0.81 & 0.48 \\
\hline Abbreviations: EPT, electrical perceptual threshold; ICC, intra-class correlation coefficients; \\
SWM, Semmes-Weinstein monofilament. \\
ICCs for each dermatome include both left and right side measures on 40 subjects (20 male, \\
20 female).
\end{tabular}

\section{Correlation between SWM and EPT}

It has been shown previously that females have marginally lower EPT values than males for some dermatomes. ${ }^{4}$ Gender difference was 
a
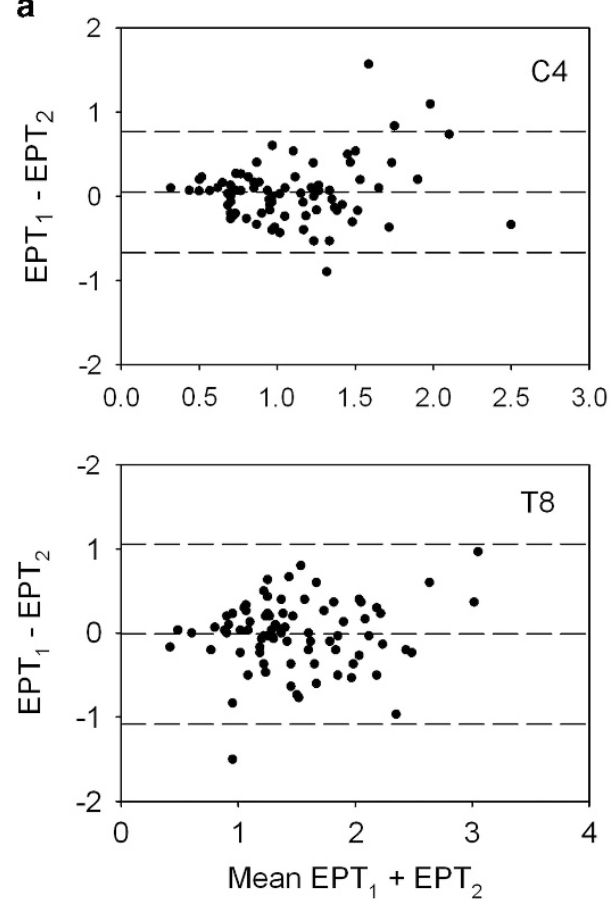

b
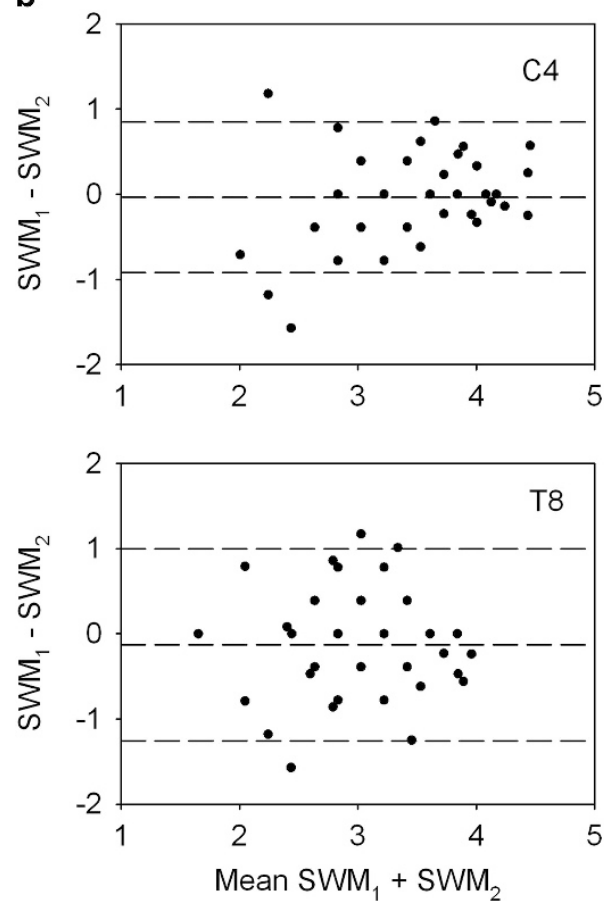
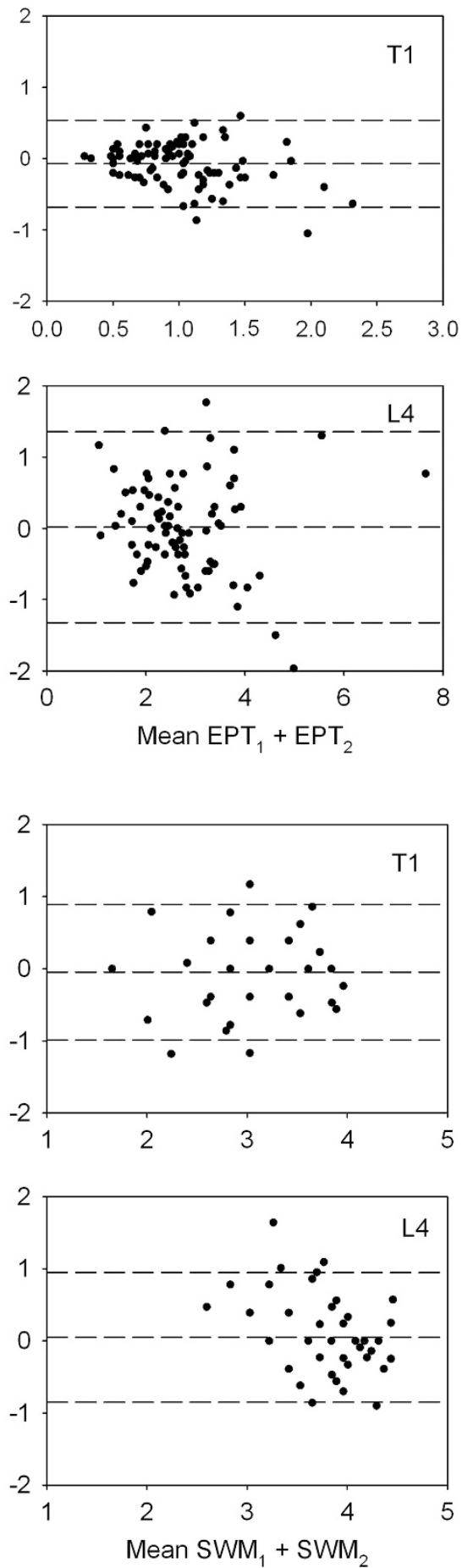

Figure 2 Bland and Altman plots for the difference between first and second (repeat) assessments against the mean value of the two assessments for (a) EPT and (b) SWM for each of the four dermatomes. Dashed lines represent mean (centre) and $95 \%$ limits (upper and lower) of the difference between assessments.

re-examined here before looking for any correlation between EPT and SWM thresholds. Figure 3 shows the mean values for EPT and SWM for male and female subjects. Females had significantly lower mean EPT than males for dermatomes C4, T1 and L4, whereas they had higher mean thresholds for SWM for dermatomes T1 and L4. As a result of identifying these gender differences, correlations between SWM and EPT were sought separately for male and female subjects. Pearson product moment correlation coefficients were insignificant for all but the L4 dermatome, which showed a weak positive correlation between the two measures for male subjects $(r=0.39, P=0.01)$. Figure 4 shows regression plots for the L4 dermatome. 

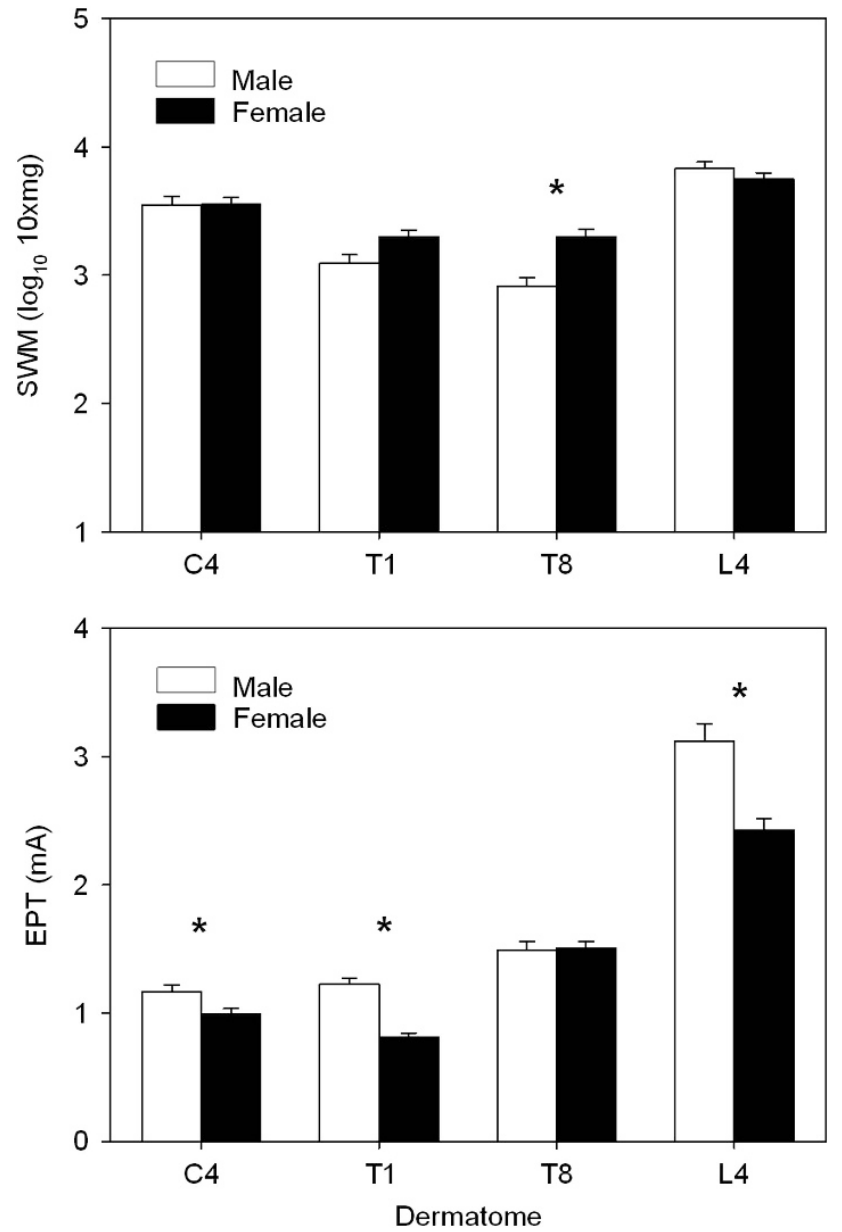

Figure 3 Mean (+s.e.) values for SWM (above) and EPT (below) thresholds for male (open bars) and female (filled bars) assessments for the four dermatomes C4, T1, T8 and L4. Significant differences $(P<0.05)$ between male and female subjects are marked with an asterisk.

\section{DISCUSSION}

The main aim of this study was to compare the reliability of the EPT and SWM tests of cutaneous sensibility in a neurologically healthy population. This was undertaken as both are quantitative measures that have the potential to add sensitivity to the AIS sensory evaluation of a SCI. Four dermatomes (C4, T1, T8, L4) widely distributed along the vertebral axis were tested. Results from the ICC analysis showed the EPT to have moderately good repeatability (ICC $0.67-0.81$ ) compared to only a fair level of repeatability (ICC $0.46-0.61$ ) for the SWM for these dermatomes. However, as the variability in ICC values indicates repeatability for EPT and SWM as measured by the ICC may be different for other dermatomes. A higher ICC for the EPT compared to the SWM was also seen when male and female subjects were assessed separately. The ICC results for EPT are broadly in agreement with previous estimates of reliability carried out for clinically normal dermatomes well above the level of SCI (King et $\left.a .^{7}\right)$ and higher ICC (0.75-0.90) results for EPT have been found for a neurologically healthy group by Leong et al. ${ }^{8}$ For SWM, intrarater reliability in healthy individuals has been reported as wide ranging, from poor to good. ${ }^{15-17}$ Substantial reliability for SWM has been reported in $\mathrm{SCI}^{15}$ and exceptionally good reliability recorded for a very wide range of sensibilities in subjects that included both Braille readers and nerve injury. ${ }^{18}$ Reliability for $\mathrm{EPT}$ in $\mathrm{SCI}^{7}$ was broadly
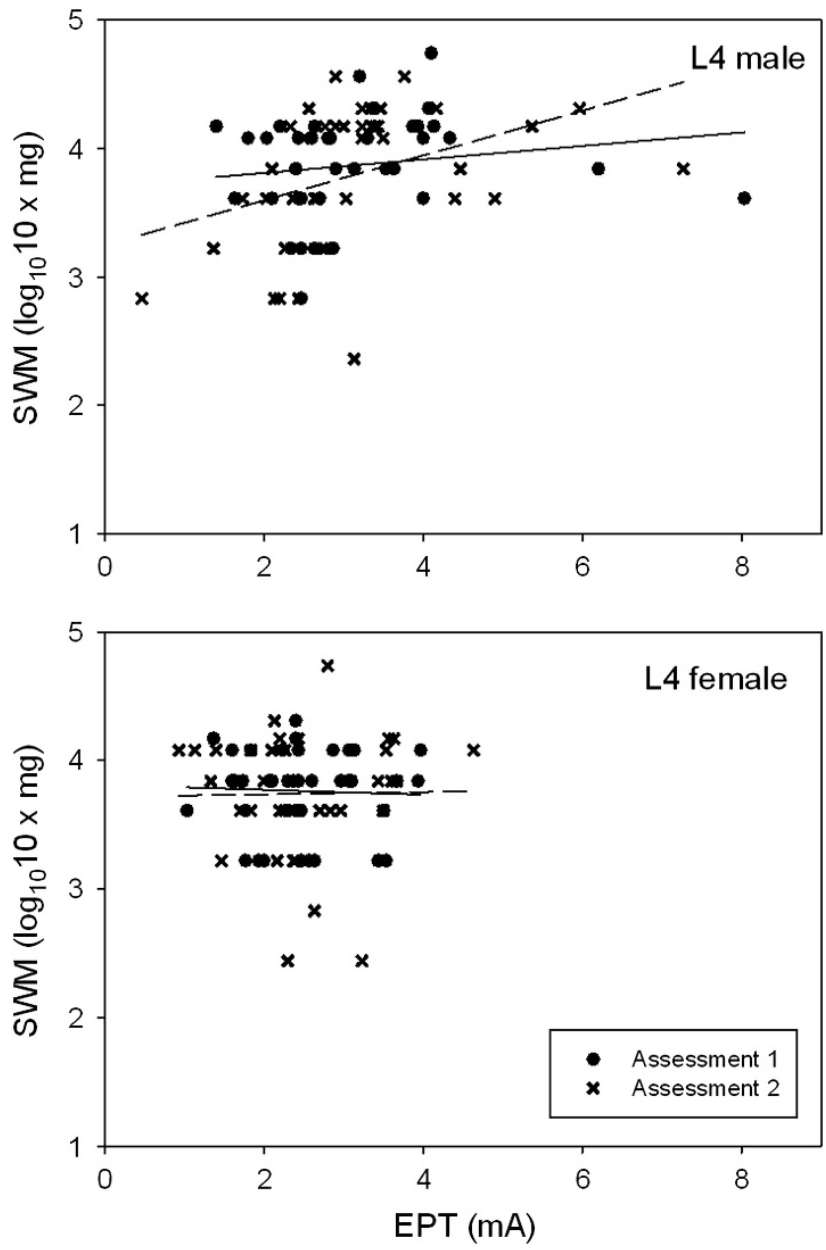

Figure 4 Regression analysis showing poor correlation between EPT and SWM tests. Data for the L4 dermatome for the first (closed circles, solid lines) and second (cross symbols, dashed lines) assessments for male (above) and female (below) subjects. Regression significant only for second assessment for male subjects $(r=0.26, P=0.02)$.

similar to that found in the present study on healthy individuals. However, in that study, the range of EPT was similar for dermatomes affected and unaffected by SCI probably due to the study having been based mainly on well-recovered ASIA D subjects with little difference in range of EPT above and below the level of injury. Where the range of sensibilities in a study is greater, it is possible, as with $\mathrm{SWM},{ }^{18}$ that ICC will indicate better reliability for EPT.

As another index of reliability, there was no evident relationship between the difference in repeated measures and the magnitude of the mean measure for either EPT or SWM. However, averaging and subtracting the SWM values for the Bland-Altman plots is an inexact process due to the logarithmic rating of the monofilaments. BlandAltman plots of the actual forces exerted by SWM showed very large increases in difference between measures as the magnitude of the force increased.

It had been anticipated that the thresholds for cutaneous sensibility measured by the EPT and SWM would be correlated. Apart from a weak correlation for one dermatome, this was not the case. The reason for this is not clear. One possibility is that the two measures test different sensory modalities. The weak electrical currents that determine EPT would have stimulated the largest myelinated axons that innervate rapidly adapting receptors ${ }^{19}$ and those tend to have the 
lowest mechanical thresholds. ${ }^{20}$ The SWM test requires persistent pressure until the monofilament bends, so such stimulation may be exciting slowly adapting receptors that give a maintained discharge to pressure. These have smaller diameter axons with higher electrical thresholds. An alternative reason for the lack of correlation may be that this study examined a healthy population with a relatively narrow range of cutaneous sensitivity. Extending this study to SCI subjects with impaired, and thus a wider range of cutaneous sensibility might be expected to reveal a significant correlation between EPT and SWM. This is not certain; however, as the level of agreement in SCI between SWM and light touch as measured by the AIS is itself not substantial. ${ }^{14}$

In conclusion, this study reveals EPT to have better reliability than the SWM test, but indicates that the two tests may be assessing different modalities of cutaneous sensibility. Thus, the improvement in sensitivity required for the quantitative assessment of the sensory status of an individual with SCI ahead of recuperative trials might benefit from the application of both tests. As a next step, this study suggests that the reliability of both EPT and SWM should be assessed together in a stable SCI population with a wide range of level and severity of SCI.

\section{DATA ARCHIVING}

There were no data to deposit.

\section{CONFLICT OF INTEREST}

The authors declare no conflict of interest.

\section{ACKNOWLEDGEMENTS}

Supported by the US Army Medical Research and Materiel Command and the Christopher Reeve Foundation.

1 Marino RJ, Barros T, Biering-Sorensen F, Burns SP, Donovan WH, Graves DE et al. ASIA Neurological Standards Committee 2002. International standards for neurological classification of spinal cord injury. J Spinal Cord Med 2003; 26: S50-S56.

2 Steeves JD, Lammertse D, Curt A, Fawcett JW, Tuszynski MH, Ditunno JF et al. International campaign for cures of spinal cord injury paralysis. Guidelines for the conduct of clinical trials for spinal cord injury $(\mathrm{SCI})$ as developed by the ICCP panel: clinical trial outcome measures. Spinal Cord 2007; 45: 206-221.

3 Hayes KC, Wolfe DL, Hsieh JT, Potter PJ, Krassioukov A, Durham CE. Clinical and electrophysiologic correlates of quantitative sensory testing in patients with incomplete spinal cord injury. Arch Phys Med Rehabil 2002; 83: 1612-1619.

4 Davey NJ, Nowicky AV, Zaman R. Somatopy of perceptual threshold to cutaneous electrical stimulation in man. Exp Physiol 2001; 86: 127-130.

5 Ellaway PH, Anand P, Bergstrom EM, Catley M, Davey NJ, Frankel HL et al. Towards improved clinical and physiological assessments of recovery in spinal cord injury: a clinical initiative. Spinal Cord 2004; 42: 325-337.

6 Savic G, Bergström EM, Frankel HL, Jamous MA, Ellaway PH, Davey NJ. Perceptual threshold to cutaneous electrical stimulation in patients with spinal cord injury. Spinal Cord 2006; 44: 560-5666.

7 King NK, Savic G, Frankel H, Jamous A, Ellaway P. Reliability of cutaneous electrical perceptual threshold in the assessment of sensory perception in patients with spinal cord injury. J Neurotrauma 2009; 26: 1061-1068.

8 Leong GW, Gorrie CA, Ng K, Rutkowski S, Waite PM. Electrical perceptual threshold testing: a validation study. J Spinal Cord Med 2009; 32: 140-146.

9 Kramer JL, Moss AJ, Taylor P, Curt A. Assessment of posterior spinal cord function with electrical perception threshold in spinal cord injury. J Neurotrauma 2008; 25: 1019-1026.

10 Savic G, Frankel HL, Jamous MA, Jones PW, King NK. Sensitivity to change of the cutaneous electrical perceptual threshold test in longitudinal monitoring of spinal cord injury. Spinal Cord 2011; 49: 439-444.

11 Ellaway PH, Kuppuswamy A, Balasubramaniam AV, Maksimovic R, Gall A, Craggs MD et al. Development of quantitative and sensitive assessments of physiological and functional outcome during recovery from spinal cord injury: a clinical initiative. Brain Res Bull 2011; 84: 343-357.

12 Finnerup NB, Sørensen L, Biering-Sørensen F, Johannesen IL, Jensen TS. Segmental hypersensitivity and spinothalamic function in spinal cord injury pain. Exp Neurol 2007; 207: 139-149.

13 Nicotra A, Ellaway PH. Thermal perception thresholds: assessing the level of human spinal cord injury. Spinal Cord 2006; 44: 617-624.

14 Kalsi-Ryan S, Beaton D, Curt A, Duff S, Popovic MR, Rudhe C et al. The graded redefined assessment of strength sensibility and prehension (GRASSP)—reliability and validity. J Neurotrauma 2012; 29: 905-914.

15 Rozental TD, Beredjiklian PK, Guyette TM, Weiland AJ. Intra- and interobserver reliability of sensibility testing in asymptomatic individuals. Ann Plast Surg 2000; 44: 605-609.

16 Felix ER, Widerstrom-Noga EG. Reliability and validity of quantitative sensory testing in persons with spinal cord injury and neuropathic pain. J Rehabil Res Develop 2009; 46: $69-84$.

17 Collins S, Visscher P, de Vet HC, Zuurmond WWA, Perez RSGM. Reliability of the Semmes-Weinstein monofilaments to measure cutaneous sensibility in the feet of healthy subjects. Disability Rehabil 2010; 32: 2019-2027.

18 Novak CB, Mackinnon SE, Williams JI, Kelly L. Establishment of reliability in the evaluation of hand sensibility. Plast Reconstr Surg 1993; 92: 311-322.

19 Brown AG, Iggo A. A quantitative study of cutaneous receptors and afferent fibres in the cat and rabbit. J Physiol 1967; 193: 707-733.

20 Johansson RS, Vallbo AB, Westling G. Thresholds of mechanosensitive afferents in the human hand as measured with von Frey hairs. Brain Res 1980; 184: 343-351. 